\title{
Gamma-Delta CAR-T Cells Show CAR-Directed and Independent Activity Against Leukemia
}

\begin{abstract}
Meir Rozenbaum ${ }^{1,2,3}$, Amilia Meir ${ }^{3}$, Yarden Aharony ${ }^{3}$, Orit Itzhaki², Jacob Schachter ${ }^{2}$, Ilan Bank ${ }^{4}$, Elad Jacoby ${ }^{3,5,6 *}$ and Michal J. Besser ${ }^{1,2,7 *}$

${ }^{1}$ Department of Clinical Microbiology and Immunology, Sackler School of Medicine, Tel Aviv University, Tel Aviv, Israel, ${ }^{2}$ Ella Lemelbaum Institute for Immuno Oncology, Sheba Medical Center, Ramat Gan, Israel, ${ }^{3}$ Center for Pediatric Cell Therapy, Sheba Medical Center, Tel Hashomer, Israel, ${ }^{4}$ Rheumatology Unit, Sheba Medical Center, Tel Hashomer, Israel, ${ }^{5}$ Division of Pediatric Hematology and Oncology, Sheba Medical Center, The Edmond and Lily Safra Children's Hospital, Tel Hashomer, Israel, ${ }^{6}$ Department of Pediatrics, Sackler School of Medicine, Tel Aviv University, Tel Aviv, Israel, ${ }^{7}$ Wohl Institute of

Translational Medicine, Sheba Medical Center, Tel Aviv, Israel
\end{abstract}

Autologous T cells engineered to express a chimeric antigen receptor (CAR) against the CD19 antigen are in the frontline of contemporary hemato-oncology therapies, leading to high remission rates in B-cell malignancies. Although effective, major obstacles involve the complex and costly individualized manufacturing process, and CD19 target antigen loss or modulation leading to resistant and relapse following CAR therapy. A potential solution for these limitations is the use of donor-derived $\gamma \delta T$ cells as a CAR backbone. $\gamma \delta T$ cells lack allogenecity and are safely used in haploidentical transplants. Moreover, $\gamma \delta T$ cells are known to mediate natural anti-tumor responses. Here, we describe a 14-day production process initiated from peripheral-blood mononuclear cells, leading to a median 185-fold expansion of $\gamma \delta$ T cells with high purity (>98\% CD3+ and $>99 \%$ $\gamma \delta \mathrm{TCR}+$ ). CAR transduction efficacy of $\gamma \delta \mathrm{T}$ cells was equally high when compared to standard CAR-T cells (60.5 \pm 13.2 and $65.3 \pm 18.3 \%$, respectively). CD19-directed $\gamma \delta$ CAR-T cells were effective against CD19+ cell lines in vitro and in vivo, showing cytokine production, direct target killing, and clearance of bone marrow leukemic cells in an NSG model. Multiple injections of $\gamma \delta C A R-T$ cells and priming of mice with zoledronate lead to enhanced tumor reduction in vivo. Unlike standard CD19 CAR-T cells, $\gamma \delta C A R-T$ cells were able to target CD19 antigen negative leukemia cells, an effect that was enhanced after priming the cells with zoledronate. In conclusion, $\gamma \delta \mathrm{CAR}-\mathrm{T}$ cell production is feasible and leads to highly pure and efficient effector cells. $\gamma \delta \mathrm{CAR}-\mathrm{T}$ cell may provide a promising platform in the allogeneic setting, and may target leukemic cells also after antigen loss.

Keywords: gamma-delta T cells, chimeric antigen receptor, leukemia, immuno oncology, B cell malignancies

\section{INTRODUCTION}

Transduction of $\mathrm{T}$ cells with a chimeric-antigen receptor (CAR) enables the targeting of extracellular domains, leading to CAR-induced T cell cytotoxicity, and cytokine production, in an MHC-independent manner (1). Autologous CAR-T cells directed against the pan-B-cell antigen CD19 have been approved for acute lymphoblastic leukemia (ALL) and non-Hodgkin lymphoma 
(NHL), after showing high remission rates in heavily pretreated patients, which in some may be durable $(2,3)$. Still, many patients do not respond to CAR-T cells or experience a relapse, through an orchestra of mechanisms including loss of transferred $\mathrm{T}$ cells in some patients and alterations in target antigen expression or density in others $(4,5)$. Thus, further multi-targeting approaches have been proposed, usually via dual CARs $(5,6)$, but sequential antigen loss has also been shown following CAR-mediated multitargeting $(7,8)$. In addition these cellular therapies are still autologous products, necessitating complex, and individualized production associated with a significant financial burden.

Lymphocytes bearing the $\gamma \delta \mathrm{T}$ cell receptor $(\gamma \delta \mathrm{T})$ are a small subset of peripheral blood cytotoxic $\mathrm{T}$ cells, which do not require antigenic presentation by MHC molecules for recognition and function $(9,10)$. In vitro and in vivo expansion of these cells is feasible, especially when exposing them to amino bisphosphonates such as zoledronate $(11,12) . \gamma \delta \mathrm{T}$ cells are known to function across MHC-barriers, and do not cause graft-vs.-host disease (13). Moreover, anti-tumor activity has been demonstrated using expanded $\mathrm{V} \gamma 9 \mathrm{~V} \delta 2 \mathrm{~T}$ cells in preclinical studies and early phase clinical trials (14), though effects against ALL and NHL remain modest at most $(13,15,16)$.

Since $\gamma \delta \mathrm{T}$ cells can be safely applied in the allogeneic setting and exhibit natural anti-tumor reactivity, arming $\gamma \delta \mathrm{T}$ cells with a CAR may provide a way to safely use allogeneic CARs and can potentially target minor clones with lower antigen density, which may not be eliminated by the standard CAR T cells. Here, we report our protocol to use $\gamma \delta \mathrm{T}$ lymphocytes as a platform for CAR-T cells. We show that $\gamma \delta$ CAR-T cells are effective against CD19 malignancies in vitro and in vivo, and have activity against leukemic clones lacking CD19 expression.

\section{MATERIALS AND METHODS}

\section{Ethics}

Patient material was obtained as part of a clinical trial previously reported (NCT02772198) $(17,18)$ and approved by the Sheba Medical Center IRB and the Israeli Ministry of Health. All animal experiments were approved by Institutional Ethical Review Process Committees and were performed under Israel Institutional Animal care and use committee approval (1131/17/ANIM).

\section{Cell Culture}

Leukemia cell lines Nalm6, CCRF-CEM, Toledo, and K562 were kindly provided by Steve Feldman, and grown in standard culture conditions, using RPMI medium supplemented with $10 \%$ fetal bovine serum (FBS), $2 \mathrm{mM}$ L-Glutamate, Sodium Pyruvate, Hepes buffer $0.1 \mathrm{M}$ (all from Biological Industries), and $100 \mathrm{U} / \mathrm{mL}$ penicillin and $100 \mu \mathrm{g} / \mathrm{mL}$ streptomycin (SigmaAldrich) ("target cell medium"). For activation and transduction, $\mathrm{T}$ cells were cultured in RPMI supplemented with $10 \% \mathrm{FBS}$, $2 \mathrm{mM} \mathrm{L}$-Glu, $100 \mathrm{U} / \mathrm{mL}$ penicillin, and $100 \mu \mathrm{g} / \mathrm{mL}$ streptomycin and interleukin-2 (IL-2, $100 \mathrm{IU} / \mathrm{ml}$, Novartis Proleukin) ("T cell medium"). 293T cells used for viral production were cultivated in DMEM high glucose medium supplemented with 10\% FBS, 2 mM L-Glutamate, Sodium Pyruvate, Hepes buffer
0.1 M, and non-essential amino acids solution (all from Biological Industries). Alternatively, medium can be supplemented with human $\mathrm{AB}$ serum instead of FBS.

\section{CAR-T Cell Production}

Peripheral-blood mononuclear cells (PBMCs) were isolated using centrifugation on LymphoprepTM density gradients (Alere technologies) and were activated in $\mathrm{T}$ cell medium with 100 $\mathrm{IU} / \mathrm{ml}$ IL2 and either OKT3 $50 \mathrm{ng} / \mathrm{ml}$ (Invitrogen) for standard $\mathrm{T}$ cell expansion (previously published) (17), or zoledronic acid $2.94 \mathrm{uM}$ (Novartis) for $\gamma \delta \mathrm{T}$ cell expansion. On day 5 of culture, activated cells were transduced with the CD19 CAR retrovirus, based on an MSGV backbone transduced with an FMC63-CD28CD3zeta plasmid, kindly provided by Steve Feldman. For this purpose, non-tissue culture treated 6-well-plates (Falcon) were pre-coated with 20 ug Retronectin per well $(10 \mathrm{ug} / \mathrm{ml}$, TakaraClontech) for 3 days at $4^{\circ} \mathrm{C}$ followed by a $20 \mathrm{~min}$ incubation with a $2.5 \%$ BSA solution (Caisson labs, BSA fraction V) in PBS (Biological Industries) and a single wash with PBS. Plates were loaded with $4 \mathrm{ml}$ of virus diluted $1: 1$ with $\mathrm{T}$ cell medium per well and centrifuged at $2,000 \mathrm{~g}$ for $2 \mathrm{~h}$ at $32^{\circ} \mathrm{C}$. Following centrifugation, the supernatant was collected leaving only $1 \mathrm{ml} /$ well. Plates were then seeded with the OKT-3 or zoledronic acid activated PBMCs, between 2 and $2.5 \times 106$ cell/well, centrifuged at $1,000 \mathrm{~g}$ for $20 \mathrm{~min}$ at $32^{\circ} \mathrm{C}$ and incubated overnight at $37^{\circ} \mathrm{C}$. Un-transduced $\gamma \delta \mathrm{T}$ cells and standard T cells were treated the same way, just without addition of virus and served as negative control. On Day $9 \gamma \delta \mathrm{T}$ and $\gamma \delta \mathrm{CAR}-\mathrm{T}$ cells underwent $\alpha \beta T C R+$ cell depletion using MACS LD depletion magnetic columns, FcR Blocking reagent, anti-TCRa/b-Biotin, and anti-biotin MicroBeads (all from Miltenyi). The remaining $\gamma \delta \mathrm{T}$ cells and standard CAR-T cells were further expanded in IL-2 containing T cell medium until day 13-15.

Standard CAR T cells for the clinical setting were produced the same way as standard CAR T described here, with the only differences that clinical CAR T production utilizes $300 \mathrm{IU} / \mathrm{ml} \mathrm{IL-}$ 2 (instead of $100 \mathrm{IU} / \mathrm{ml}$ ), transduction is performed on day 2 (instead of day 5) and human $\mathrm{AB}$ serum is supplemented to the culture medium (instead of FBS) (17).

\section{Co-culture, Cytokine Levels, and Cytotoxic Assays}

Co-cultures were carried out in flat bottom 96-well-plates. For IFN- $\gamma$ secretion tests $\mathrm{T}$ cells and targets were plated 100,000 cell/well each overnight in target cell medium. ELISA for the detection of human IFN- $\gamma$ was carried out using Biolegend's Elisa $\mathrm{MAX}^{\mathrm{TM}}$ Deluxe kit. For cytotoxic assays, targets were stained by CellTrace ${ }^{\mathrm{TM}}$ Violet according to manufacturer's instructions and seeded 40,000 cells/well. T cells were then added at 160,000 cells $/ \mathrm{ml}$. After $2.5-3 \mathrm{~h}$ at $37^{\circ} \mathrm{C}$, cells were collected from wells and stained for apoptosis by Annexin V-Cy5 reagent (Biovision). CellTrace positive cells were assessed for AnnexinV staining by flow cytometry.

\section{Flow Cytometry}

Flow cytometry was performed on a Beckman Coulter's Gallios. For detection of the CD19 CAR receptor we used a biotinylated 
A

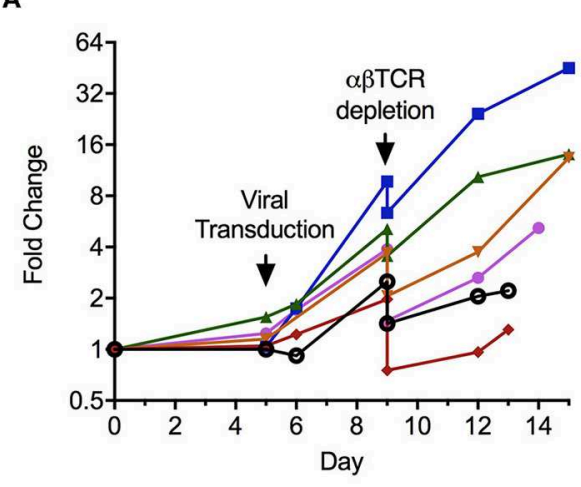

B

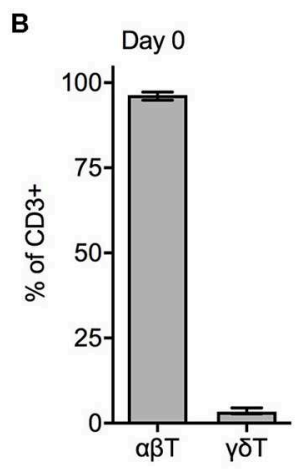

c

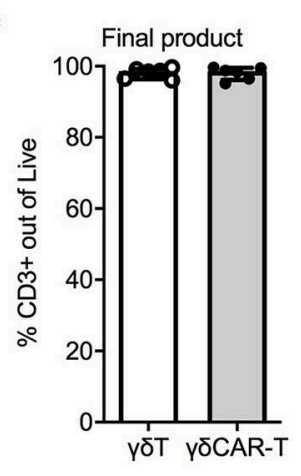

D

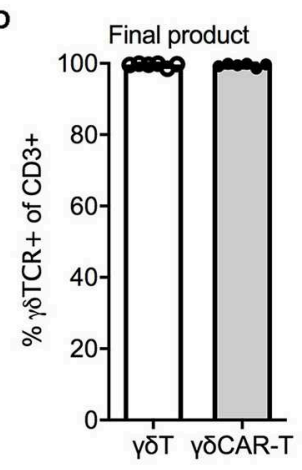

E

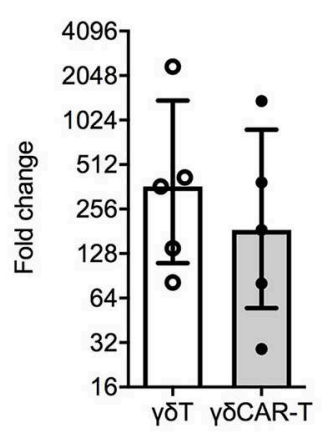

F

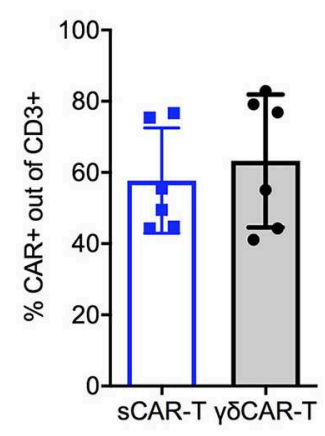

G

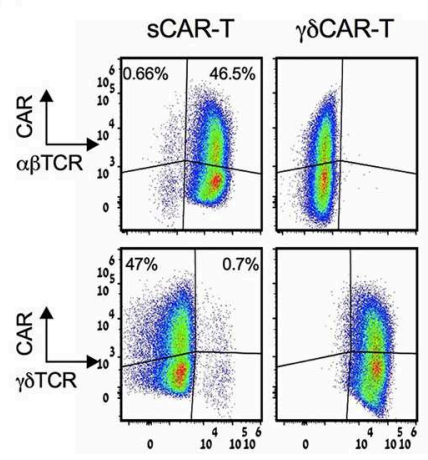

H

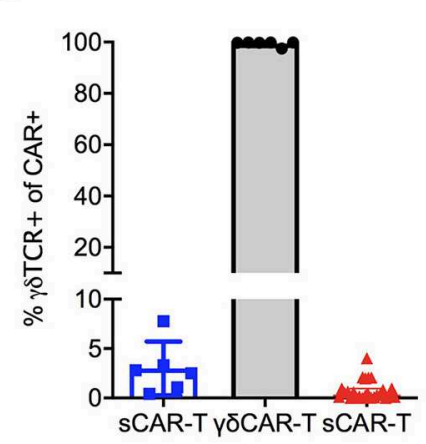

FIGURE 1 | Production of $\gamma \delta C A R-T$ cells: (A) Production scheme, showing total cell expansion (by fold change) along the protocol. Day 0 is the collection of blood, PBMCs isolation, and T cell activation. Each line represents a different healthy donor. (B) $\gamma \delta-\mathrm{T} / \alpha \beta$-T cells composition of donors' blood out of CD3 positive cells $(n=$ 5). (C) CD3 positive cells in the final product of un-transduced $\gamma \delta$-T and transduced $\gamma \delta$ CAR-T cells $(n=6)$. (D) Purity of $\gamma \delta$ TCR + cells in the final product of both protocols $(n=6)$. (E) $\gamma \delta$-T cells fold change expansion during the $\gamma \delta \mathrm{CAR}$-T production protocol $(n=5)$. (F) CAR transduction efficiency by flow cytometry gated on CD3 positive cells, of the standard CAR (sCAR) and $\gamma \delta C A R-T$ cell products $(n=6)$. (G) Dot plots of a representative sample showing CAR expression in $\gamma \delta$ and $\alpha \beta-T$ cells populations in the final product of sCAR and $\gamma \delta \mathrm{CAR}-\mathrm{T}$ cell protocols. (H) $\gamma \delta \mathrm{TCR}$ positive cells gated on CAR positive cells in the final composition of sCAR-T cells (blue squares, $n=6$ ), $\gamma \delta$ CAR-T cells production protocol (black circles, $n=6$ ) and the clinically manufactured sCAR-T cells (red triangles, $n=25)$. Bars are at the median value, and error bars represent interquartile range.

anti-mouse FAB as a primary antibody (Jackson), Mouse Gamma Globulin as a blocking reagent (Jackson) and Anti-BiotinViogreen as a secondary antibody (Miltenyi). The following antibodies were used for additional staining: Anti Human CD3 FITC, Anti Human TCRa/b-APC, Anti Human TCRg/d-PE, Anti Human CD19-PE, Anti Human CD10-PE-Cy7, Anti Human CD45 viogreen, Anti mouse CD45 APC, and for dead cell exclusion Ghost Red 780 Viability Dye (all from Miltenyi). All antibodies were incubated with samples for $20 \mathrm{~min}$ at $4^{\circ} \mathrm{C}$. Analysis was done using the FlowJo analysis software V10.

\section{Nalm6 CRISPR CD19 KO}

We generated a Lenti virus expressing CAS9 and a sgRNA targeting CD19. The sgRNA sequence 5'-TGGAATGTTTCG GACCTAGGTGG-3' (19) was cloned into pL-CRISPR.EFS.GFP (addgene Plasmid \#57818 - Lentiviral CRISPR-Cas9 delivery for SpCas9 and sgRNA. Co-expresses eGFP via P2A cleavage site). Envelope plasmid was pMD2.G (addgene Plasmid \#12259) and packaging plasmid psPAX2 (addgene Plasmid \#12260). Plasmids were transfected to $293 \mathrm{~T}$ cell line using the calcium Phosphate Profection Mammalian Transfection System
(Promega cat\#E1200). Viral supernatant was used for infection of Nalm6 cells with addition of Polybrene $(8 \mathrm{ng} / \mathrm{ml})$. CD19 positive cell were depleted using PE conjugated anti-CD19 antibody and anti-PE magnetic beads, on a MACS LD depletion column (Miltenyi), followed by single cell culture by plating. CD19 negative single-clones were confirmed by flow cytometry and wells were sequenced for the CD19 KO locus.

\section{Animals and in vivo Models}

For all in vivo experiments, 8-15-weeks-old NOD-SCID-IL-2R $\gamma$ (NSG) female mice, purchased from the Jackson laboratories, were used. Mice were tail-vein injected with $1 \times 10^{6} \mathrm{Nalm} 6$ cells for leukemia inoculation, followed by intravenous (IV) injections of effector cells with or without intraperitoneal (IP) injections of zoledronate (20).

\section{Statistical Analysis}

All statistical analyses were performed using the Prism v8 (GraphPad Software). Statistical comparisons between two groups were determined by two-tailed parametric or nonparametric (Mann-Whitney $U$-test) $t$-tests for unpaired data 


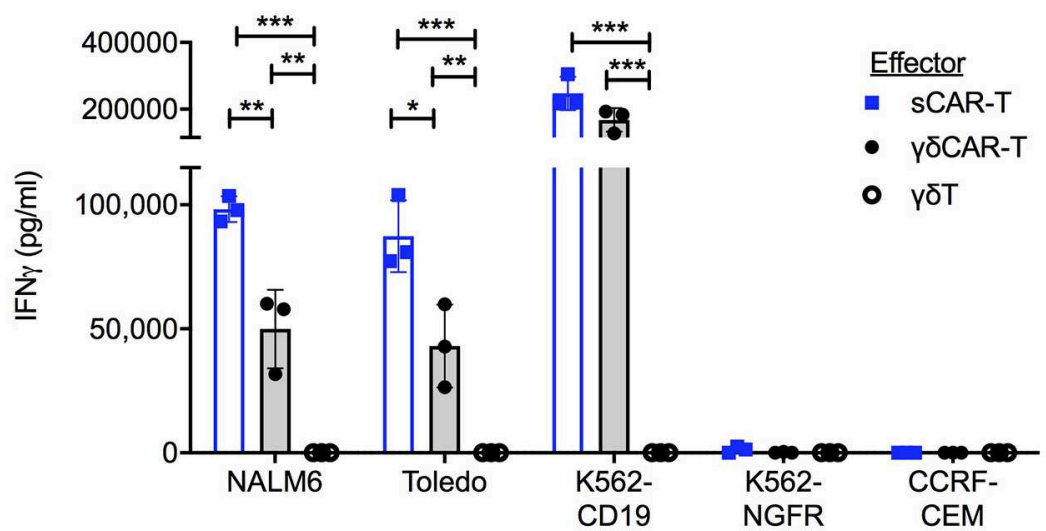

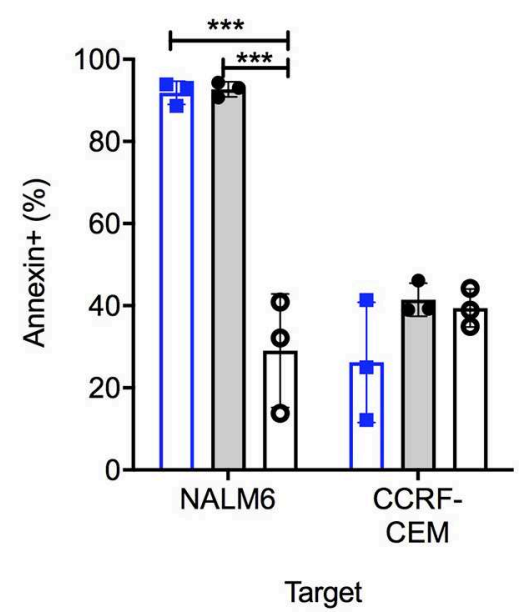

FIGURE 2 | $\gamma \delta$ CAR-T cells demonstrate CD19 target dependent activity. (A) IFN $\gamma$ levels in co-culture medium of listed effectors with CD19 positive and negative target cells. (B) Cytotoxic activity of effector cells against CD19 positive Nalm6 cells and CD19 negative CCRF-CEM cells. Cell trace violette pre-stained targets were evaluated for Annexin binding by FACS analysis. Blue bars, SCAR; Gray bars, $\gamma \delta$ CAR-T; White bars, $\gamma \delta-T$ cells. ${ }^{\star} P \leq 0.05$, ${ }^{\star \star} P \leq 0.01$, ${ }^{\star \star \star} P \leq 0.001$, Two tailed paired $T$-test. Error bars represent standard deviation.

or by two-tailed paired Student's $t$-tests for matched samples (produced from same donor). $P<0.05$ were considered statistically significant.

\section{RESULTS}

\section{Generation of Human CD19 CAR Expressing $\gamma \delta \mathrm{T}$ Cells From Peripheral Blood}

We first devised and calibrated a protocol for the generation of $\gamma \delta \mathrm{T}$ cells expressing the CD19 CAR and depletion of $\alpha \beta$-TCR + cells (Figure 1A). On average, $\gamma \delta \mathrm{T}$ cells consisted of $3.4 \%( \pm$ $0.73 \%$ ) of CD3-positive cells in the initial starting material of PBMCs (Figure 1B). Activation with Zoledronate on day 0 led to specific proliferation of $\gamma \delta \mathrm{T}$ cells, whilst the total number of cell remained similar. On Day 5, cells were transduced with the CD19 CAR, followed by $\alpha \beta$ TCR + depletion on day 9 and further proliferation of $\gamma \delta C A R-T$ for a total of $13-15$ days. Untransduced $\gamma \delta \mathrm{T}$ cells served as control. The final products of transduced as well as un-transduced $\gamma \delta \mathrm{T}$ cells contained 98\% $( \pm 1.77 \%)$ and $98.1 \%( \pm 1.49 \%)$ CD3 positive cells, respectively, with high purity of $\gamma \delta \mathrm{T}$ cells, accounting for $99.5 \%( \pm 0.5 \%)$ of the CD3 + cells $(n=6$, Figures 1C,D). The median fold change of the $\gamma \delta$ T cells was 185 (range, 29-1,376) for transduced cells compared with a median of 363 (range, 81-2,350) for untransduced $\gamma \delta \mathrm{T}$ cells, with a variable range between different donors (Figure 1E, $p=0.2$ paired $T$-test). As controls, we ran in parallel a standard CAR-T cell (sCAR-T) production for each donor, using a protocol as for the clinical setting (17), just in a smaller scale and transduction performed on day 5 instead of 2. CAR transduction efficacy ranged between 40 and $80 \%$ (Figure 1F), and did not differ between sCAR-T and $\gamma \delta C A R-T$
$(60.5 \% \pm 13.2$ and $65.3 \% \pm 18.3$, respectively). A representative FACS dot plot is presented in Figure 1G. Of note, the sCAR-T cell product had also $\gamma \delta \mathrm{T}$ cells and $\gamma \delta \mathrm{CAR}-\mathrm{T}$ cells (Figures $\mathbf{1 G}, \mathbf{H}$ ). We analyzed 25 infusion products administered to leukemia and lymphoma patients enrolled on a clinical trial for $\gamma \delta$ TCR expression. Final products had an average of $1.15 \% \gamma \delta \mathrm{T}$ and $0.77 \% \gamma \delta \mathrm{CAR}-\mathrm{T}$ (Figure 1G). The, percentage of $\gamma \delta \mathrm{CAR}-\mathrm{T}$ in the final product was not associated with response (17). To summarize, using this protocol we are able to produce a pure fraction of $\gamma \delta \mathrm{T}$ cells lacking $\alpha \beta \mathrm{T}$ cells, with high expression of the CD19 CAR, and this within a course of 2 weeks.

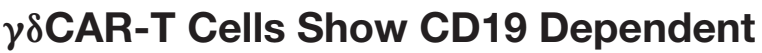 Activity Against Tumor Cell Lines}

To test for the efficacy of the $\gamma \delta \mathrm{CAR}-\mathrm{T}$ cells in comparison to the sCAR-T cells in vitro, co-culture assays against CD19 positive and negative cell lines were performed. Un-transduced activated $\gamma \delta \mathrm{T}$ cells, $\gamma \delta \mathrm{CAR}-\mathrm{T}$ cells, and sCAR-T cell were co-incubated with the B-ALL cell line Nalm6, the B-NHL cell line Toledo, and with K562 transduced to express CD19 (K562-CD19). Antigennegative controls were the T-ALL cell line CCRF-CEM and K562-NGFR cell line. Testing for IFN $\gamma$ secretion after overnight incubation at a $\mathrm{T}$ cell/target ratio of $1: 1$ revealed $\gamma \delta \mathrm{CAR}$ $\mathrm{T}$ cells are highly reactive against CD19 expressing tumor cells (Figure 2A). $\gamma \delta$ CAR-T and sCAR-T cells exhibited substantially higher levels of CD19 dependent IFN $\gamma$ secretion relative to untransduced $\gamma \delta \mathrm{T}(\gamma \delta \mathrm{CAR}-\mathrm{T}$ vs. $\gamma \delta \mathrm{T}, p=0.005, p=0.01$, and $p$ $=0.001$; sCAR-T vs. $\gamma \delta \mathrm{T}, p<0.001, p<0.001$, and $p=0.001$, for NALM6, Toledo, and K562-CD19, respectively). The level of IFN $\gamma$ in the supernatant of co-cultures with $\gamma \delta C A R-T$ cells was lower than measured in the co-culture with sCAR-T cells in the case of Nalm6 and Toledo cell lines $(p=0.007$ and $p=0.02$, 
A

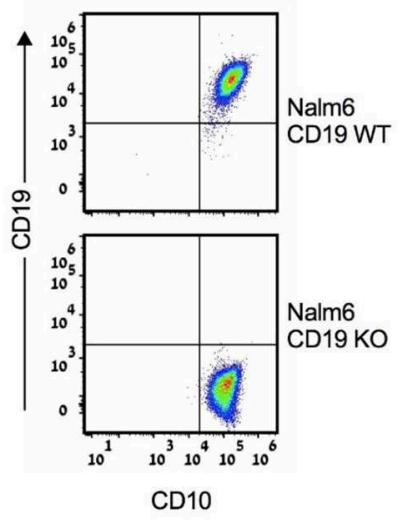

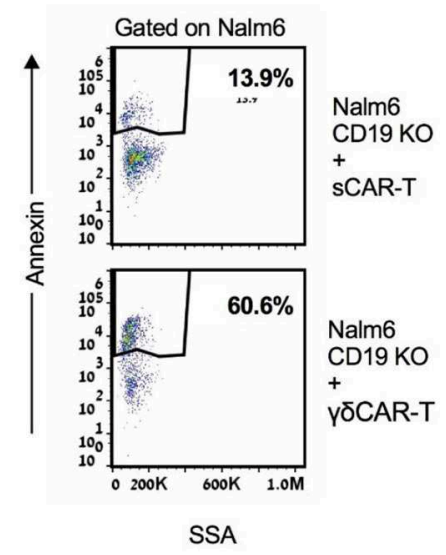

C

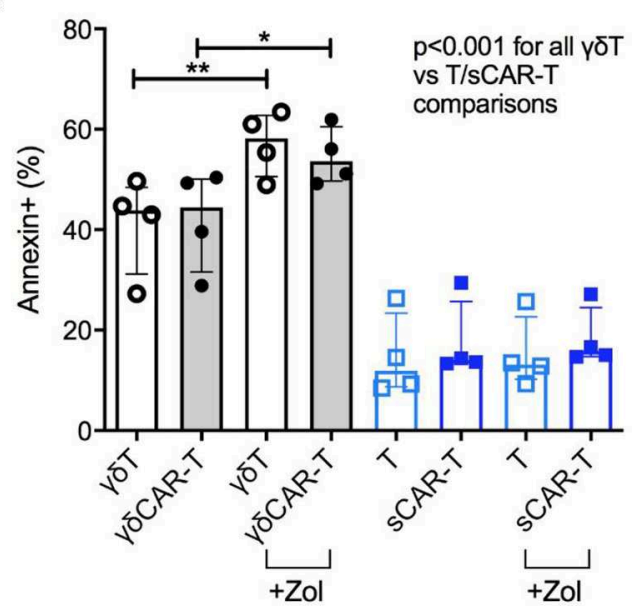

FIGURE 3 | $\gamma \delta C A R-T$ cells demonstrate activity against CD19 negative clones. (A) Generation of Nalm6 CD19 KO cells via CRISPR yields CD10 positive CD19 negative cells (Nalm6 $\left.{ }^{19 n e g}\right)$. (B,C) Activity of $\gamma \delta C A R-T$ cells against Nalm6 ${ }^{19 n e g}$ cells is further enhanced by priming the target cells with zoledronate. (B) Dot plot representation showing Annexin staining of targets cells (cell-trace violet stained Nalm6 ${ }^{19 n e g}$ ) co-cultured with sCAR-T cells (top) or $\gamma \delta C A R-T$ cells (bottom). (C) Specific cytotoxicity of different effectors against Nalm6 ${ }^{1 \text { neg }}$ cells. $\gamma \delta$-T and $\gamma \delta C A R-T$ cells in gray bars, sT, and sCAR-T cells in blue bars. Un-transduced cells in unstained circles and CAR-T cells in filled circles. ${ }^{\star} P \leq 0.05,{ }^{\star \star} P \leq 0.01$, Two tailed paired $T$-test. Error bars represent interquartile range.

respectively), but not with the artificially-expressing K562-CD19 cell line $(p=0.08$, Figure $2 \mathrm{~A})$.

In order to test for cytotoxic activity, targets were evaluated for annexin staining after a co-culture for $2.5 \mathrm{~h}$ at a $\mathrm{T}$ cell/target ratio of 5:1. Both $\gamma \delta$ CAR-T and sCAR-T cells demonstrate enhanced cytotoxic activity against the CD19 positive Nalm6, leading to $92 \%( \pm 2.8 \%)$, and $93 \%( \pm 1.8 \%)$ annexin expression on targets in comparison to activated $\gamma \delta$ T cells $(29 \pm 13.8 \%, n=3, p<0.001)$. No difference was seen in annexin expression of CD19 negative targets (Figure 2B).

\section{$\gamma \delta$ CAR-T Cells Show Enhanced in vitro Cytotoxicity Against CD19 Negative Target Cells}

A major barrier of CAR-T therapy is loss of target antigen, by various mechanisms. $\gamma \delta \mathrm{T}$ cells are known to exert an antileukemic activity, especially after priming with zoledronate (13). Thus, we hypothesized that utilizing non-specific mechanisms may result in cytotoxicity also against CD19-negative targets. To further investigate this finding, we generated a CD19 knockout B-ALL cell line Nalm6 (Figure 3A). $\gamma \delta \mathrm{CAR}-\mathrm{T}, \gamma \delta \mathrm{T}$, sCAR$\mathrm{T}$, and un-transduced activated $\mathrm{T}$ cells were co-cultured with Nalm $6^{19 n e g}$ for a period of $3 \mathrm{~h}$ at a T cell:target ratio of $4: 1$. Both $\gamma \delta \mathrm{T}$ cells and $\gamma \delta \mathrm{CAR}-\mathrm{T}$ cells demonstrated enhanced cytotoxicity against Nalm $6^{19 n e g}$ cells compared to standard T cells or sCAR$\mathrm{T}$ cells (Figures 3B,C, $p<0.001$ for all comparisons of $\gamma \delta \mathrm{T}$ or $\gamma \delta$ CAR-T vs. un-transduced $\mathrm{T}$ or sCAR-T). Priming with Zoledronate further enhanced this effect, leading to higher target annexin expression of Nalm $6^{19 n e g}$ cells incubated with $\gamma \delta \mathrm{T}(57 \%$ after priming vs. $41 \%$ without zoledronate, $p=0.007$ ) as well as $\gamma \delta \mathrm{CAR}-\mathrm{T}$ ( $55 \%$ after priming vs. $42 \%$ without zoledronate, $p$
$=0.02$, Figure $3 \mathrm{C}$ ), eluding to a potential effect that may target minor CD19-negative clones.

\section{$\gamma \delta$ CAR-T Cells Exhibit in vivo Activity Against Tumor Cell Lines}

To investigate the in vivo efficacy of $\gamma \delta C A R-T, N S G$ mice were tail-vein injected with $1 \times 10^{6}$ Nalm6 cells, and treated on day 2 with either $4 \times 10^{6} \gamma \delta$ CAR-T cells, sCAR-T, or $\gamma \delta \mathrm{T}$ cells per mouse. CAR transduction efficacy was $75-85 \%$ in all experiments. Non-treated leukemia-bearing mice served as controls. Two weeks after the injection of effector cells, mice were sacrificed for evaluation of leukemic involvement of the bone marrow. The leukemic burden in the bone marrow of non-treated mice and of $\gamma \delta \mathrm{T}$ cell treated mice was substantial (a median of 62 and 55\% of bone marrow cells, respectively). The presence of human un-transduced $\gamma \delta \mathrm{T}$ cells did not reduce significantly leukemia in this model ( $p=0.89$, Mann-Whitney). Treatment with either $\gamma \delta$ CAR-T or sCAR-T cells lead both to a drastic reduction in the leukemic burden in the bone marrow of recipient mice (to 5 and $0.1 \%$, respectively, $p<0.001$ compared to untreated or $\gamma \delta \mathrm{T}$ cell treated mice), demonstrating in vivo activity of $\gamma \delta \mathrm{CAR}-\mathrm{T}$ cells (Figure 4A). Nevertheless, traces of leukemia were noted to be higher in recipients of $\gamma \delta$ CAR-T cells in comparison with the sCAR-T treated mice. In an intent to further improve the in vivo anti-tumor reactivity of $\gamma \delta \mathrm{CAR} T$ cells, mice were conditioned with zoledronate, known to prime targets of $\gamma \delta \mathrm{T}$ cells, and a second dose of $\gamma \delta \mathrm{CAR}-\mathrm{T}$ cells was administrated, both previously shown to improve anti-tumor effect of un-transduced $\gamma \delta \mathrm{T}$ cells (21). Intraperitoneal injection of zoledronate ( $1.0 \mathrm{ug} / \mathrm{gr}$ ) to leukemia-bearing mice on days -1 , $2,6,8$, and 10 , still resulted in a median of $3 \%$ remaining leukemic cells in the bone marrow (Figure 4B). However, adding a second 


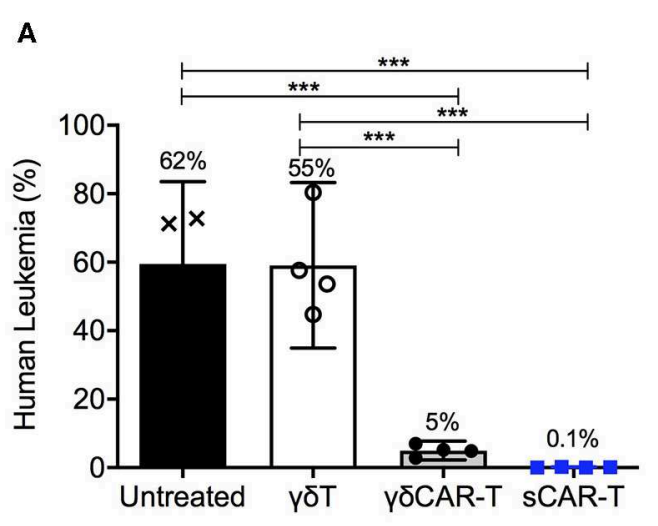

B

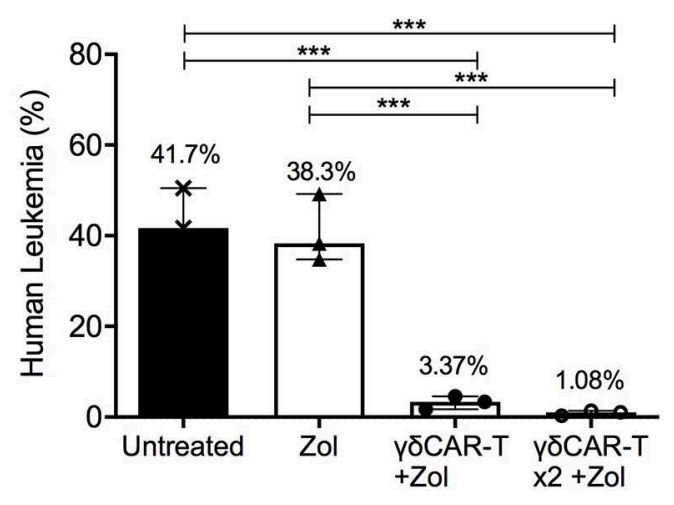

c
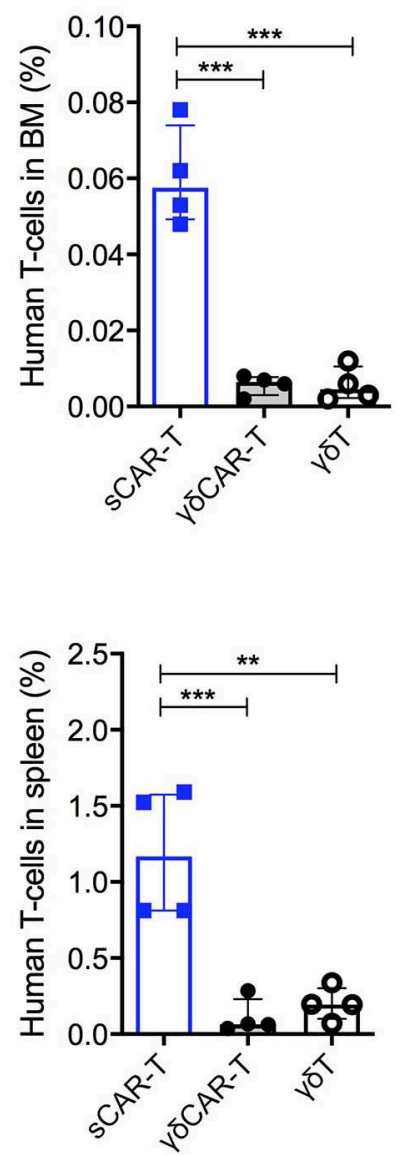

FIGURE 4 | In vivo activity of $\gamma \delta$ CAR-T cells. (A) Human leukemia (Nalm6) gated on CD45+ CD10+ in the bone marrow of NSG-mice either untreated or treated with un-transduced $\gamma \delta$ T cells, $\gamma \delta C A R-T$, or sCAR-T cells after 14 days. (B) Human leukemia (Nalm6) gated on CD45+ CD10+ in the bone marrow of NSG-mice either untreated or treated with zoledronate (Zol) alone, Zol with $\gamma \delta C A R T$ cells, or repeated dose of Zol and $\gamma \delta C A R-T$. (C) Human effector cell persistence of.i.v. injected sCAR-T, $\gamma \delta$ CAR-T, and un-transduced $\gamma \delta$-T cells in mice bone marrow (upper panel) and spleen (lower panel), 3 days post injection. ${ }^{\star \star} P \leq 0.01$, ${ }^{\star \star \star} P \leq 0.001$, Two tailed paired $T$-test. Bars are at the median value, and error bars represent interquartile range.

dose of $\gamma \delta$ CAR-T cells on day 7, $3 \times 10^{6} \mathrm{~T}$ cells per mouse, in addition to zoledronate administration regimen lead to further reduction in Nalm6 burden in the marrow to almost $1 \%(p=$ 0.06 , Figure 4B).

Since early loss of effector $\mathrm{T}$ cells may be associated with target-positive relapse, we next tested the persistence of the infused effector cells within the mice. Cell-trace violet stained $\gamma \delta \mathrm{T}, \gamma \delta \mathrm{CAR}-\mathrm{T}$, and sCAR-T cells $\left(5 \times 10^{6}\right.$ per mouse $)$ were injected to leukemic mice. After 3 days, bone marrow and spleen were harvested and tested for presence of dye positive cells (Figure 4C). Lower number of human effector T cells were found in both the spleen and the bone marrow of murine recipients of $\gamma \delta$ CAR-T and $\gamma \delta$ T cells compared to recipients of sCAR-T cells $(p$ $<0.001$ for both comparisons in the bone marrow and $p<0.01$ for both comparisons in the spleen). Thus, in the NSG model, we noted good anti-leukemic activity but limited persistence of $\gamma \delta$ CAR-T cells.

\section{DISCUSSION}

In this work we demonstrated the ability to effectively transduce and expand $\gamma \delta$ T cells with a CAR targeting CD19. $\gamma \delta$ CAR-T were effective against CD19+ tumor cell lines, both in vivo and in vitro. Moreover, we could see an effect against CD19- clones, which was CAR-independent.

Lack of allogenicity and potential of 3rd party-use make $\gamma \delta$ cells excellent candidates as a CAR-T cell backbone. Attempts of $\gamma \delta \mathrm{T}$ cell transduction with CARs have been previously reported, via zoledronate-based expansion of 1st-generation CARs (15) or proliferation of polyclonal $\gamma \delta \mathrm{T}$ cells transduced with CD19 on an antigen-presenting cells (22). Both methods include a selection process, to ensure purity of the $\gamma \delta \mathrm{T}$ cells, similar to our protocol. Of note, both methods showed in vitro efficacy against CAR target, but this was not compared to standard CAR$\mathrm{T}$ cells, which in current days of commercially available CAR-T 
cells is essential. We preformed head-to-head comparison of the $\gamma \delta$ CAR-T to sCAR-T, showing comparable transduction efficacy and in vitro cytotoxicity against CD19 positive targets, and an in vivo effect that was profound, but inferior to that of sCAR-T. We also demonstrated the presence of $\gamma \delta \mathrm{CAR}-\mathrm{T}$ cells in clinical products, though at low percentages. The Sadelain group has previously showed comparable activity of $\gamma \delta C A R-T$ and sCAR$\mathrm{T}$ in vitro and in vivo, against an intraperitoneal Raji tumor model (23). Raji, a Burkitt-NHL cell line, is known to express co-stimulatory molecules (24), which may have assisted in vivo killing by the $\gamma \delta$ CAR-T cells. Similar to others (22), we too could not show complete clearance of the aggressive Nalm6 ALL cell line in murine models treated with $\gamma \delta \mathrm{CAR}-\mathrm{T}$ cells, which might be explained by the limited persistence of $\gamma \delta \mathrm{CAR}-\mathrm{T}$ cells in comparison to sCAR-T cells. Indeed repeated infusion of the $\gamma \delta$ CAR-T improved anti-leukemic results. Loss of $\gamma \delta$ CAR-T may be a result of several factors, including lack of a supporting microenvironment for these cells in an immune-suppressed mouse, which may be improved with cytokine supplementation such as IL-2 (25). Another possible contribution may be due to increased activation-induced cell death (AICD) known to occur with activated $\gamma \delta \mathrm{T}$ cells. These challenges should be addressed in further work.

The loss of CD19 is a major problem in relapsed patients with persisting CAR-T cells, or patients with prior CD19directed therapy (26). Current models of using two (or more) CARs transduced on a single cell (by various methods) (27) show some success in preclinical models, but clinical results have not yet matured. Also, sequential antigen loss has been shown in patients with NHL or ALL. Utilizing the natural non-MHC restricted targeting optional by $\gamma \delta \mathrm{T}$ cells has shown anti-leukemic activity, which can be enhanced after priming with zoledronate (13). We showed that this effect is retained after CAR transduction of $\gamma \delta \mathrm{T}$ cells, and is independent of CAR activation by its ligand, as demonstrated by targeting antigen-negative cells. Thus, exploiting this non-specific MHCindependent targeting mechanism on top of the CAR specificity may prevent antigen loss and subsequent relapse and requires further investigation.

\section{REFERENCES}

1. June CH, Sadelain M. Chimeric antigen receptor therapy. N Engl J Med. (2018) 379:64-73. doi: 10.1056/NEJMra1706169

2. Jacoby E, Shahani SA, Shah NN. Updates on CAR T-cell therapy in B-cell malignancies. Immunol Rev. (2019) 290:39-59. doi: 10.1111/imr.12774

3. Salter AI, Pont MJ, Riddell SR. Chimeric antigen receptor modified T cells: CD19 and the road beyond. Blood. (2018) 131:2621-9. doi: 10.1182/blood-2018-01-785840

4. Hamieh M, Dobrin A, Cabriolu A, van der Stegen SJC, Giavridis T, MansillaSoto J, et al. CAR T cell trogocytosis and cooperative killing regulate tumour antigen escape. Nature. (2019) 568:112-6. doi: 10.1038/s41586-019-1054-1

5. Shah NN, Fry TJ. Mechanisms of resistance to CAR T cell therapy. Nat Rev Clin Oncol. (2019) 16:372-85. doi: 10.1038/s41571-019-0184-6

6. Perna F, Berman SH, Soni RK, Hendrickson RC, Brennan CW, Sadelain M. Integrating proteomics and transcriptomics for systematic combinatorial chimeric antigen receptor therapy of AML article integrating proteomics and transcriptomics for systematic combinatorial chimeric
In conclusion, we established a rapid and robust protocol for $\gamma \delta$ CAR-T cell production. These cells demonstrated antitumor activity in vitro and in vivo. $\gamma \delta \mathrm{CAR}-\mathrm{T}$ cell may provide a promising platform in the allogeneic setting, and may target antigen-negative clones. Further challenges, including improving in vivo persistence, are to be addressed prior to clinical application. The equations should be inserted in editable format from the equation editor.

\section{DATA AVAILABILITY STATEMENT}

The raw data supporting the conclusions of this article will be made available by the authors, without undue reservation.

\section{ETHICS STATEMENT}

The animal study was reviewed and approved by institutional ethical review process committee.

\section{AUTHOR CONTRIBUTIONS}

$\mathrm{MR}, \mathrm{EJ}, \mathrm{IB}$, and $\mathrm{MB}$ contributed conception and design of the study. MR, AM, YA, and OI performed experiments and acquired the data. MR wrote the first draft of the manuscript. EJ, IB, JS, and $\mathrm{MB}$ revised it critically for important intellectual content. All authors contributed to manuscript revision, read, and approved the submitted version.

\section{FUNDING}

This work was funded by the Dotan Research Center for Hematologic Malignancies, Tel Aviv University, Israel.

\section{ACKNOWLEDGMENTS}

We would like to thank Prof. Shai Izraeli and members of his lab, Dr. Nira Bloom and Dr. Victoria Marcu-Malina for technical assistance and fruitful discussions, and Ms. Diana Bar for assistance with patient and volunteer samples.

antigen receptor therapy of AML. Cancer Cell. (2017) 32:506-19.e5. doi: 10.1016/j.ccell.2017.09.004

7. Hegde M, Mukherjee M, Grada Z, Pignata A, Landi D, Navai SA, et al. Tandem CAR $\mathrm{T}$ cells targeting HER2 and IL13R $\alpha 2$ mitigate tumor antigen escape. J Clin Invest. (2016) 126:3036-52. doi: 10.1172/JCI 83416

8. Shalabi H, Kraft IL, Wang HW, Yuan CM, Yates B, Delbrook C, et al. Sequential loss of tumor surface antigens following chimeric antigen receptor T-cell therapies in diffuse large B-cell lymphoma. Haematologica. (2018) 103:e215-8. doi: 10.3324/haematol.2017.183459

9. Legut M, Cole DK, Sewell AK. The promise of $\gamma \delta \mathrm{T}$ cells and the $\gamma \delta \mathrm{T}$ cell receptor for cancer immunotherapy. Cell Mol Immunol. (2015) 12:656-68. doi: $10.1038 / \mathrm{cmi} .2015 .28$

10. Silva-Santos B, Serre K, Norell H. $\gamma \delta$ T cells in cancer. Nat Rev Immunol. (2015) 15:683-91. doi: 10.1038/nri3904

11. Fournié JJ, Sicard H, Poupot M, Bezombes C, Blanc A, Romagné F, et al. What lessons can be learned from $\gamma \delta$ T cell-based cancer immunotherapy trials? Cell Mol Immunol. (2013) 10:35-41. doi: 10.1038/cmi.2012.39 
12. Marcu-Malina V, Garelick D, Peshes-Yeloz N, Wohl A, Zach L, Nagar M, et al. Peripheral blood-derived, $\gamma 9 \delta 2 \mathrm{t}$ cell-enriched cell lines from glioblastoma multiforme patients exert anti-tumoral effects in vitro. J Biol Regul Homeost Agents. (2016) 30:17-30.

13. Airoldi I, Bertaina A, Prigione I, Zorzoli A, Pagliara D, Cocco C, et al. $\gamma \delta$ T-cell reconstitution after HLA-haploidentical hematopoietic transplantation depleted of TCR- $\alpha \beta+/ C D 19+$ lymphocytes. Blood. (2015) 125:2349-58. doi: 10.1182/blood-2014-09-599423

14. Deniger DC, Moyes JS, Cooper LJN. Clinical applications of gamma deltaT cells with multivalent immunity. Front Immunol. (2014) 5:636. doi: 10.3389/fimmu.2014.00636

15. Rischer M, Pscherer S, Duwe S, Vormoor J, Rossig C. Human $\gamma \delta \mathrm{T}$ cells as mediators of chimaeric-receptor redirected anti-tumour immunity. $\mathrm{Br} \mathrm{J}$ Haematol. (2004) 126:583-92. doi: 10.1111/j.1365-2141.2004.05077.x

16. Wilhelm M, Kunzmann V, Eckstein S, Reimer P, Weissinger F, Ruediger T, et al. $\gamma \delta \mathrm{T}$ cells for immune therapy of patients with lymphoid malignancies. Blood. (2003) 102:200-6. doi: 10.1182/blood-2002-12-3665

17. Itzhaki O, Jacoby E, Nissani A, Levi M, Nagler A, Kubi A, et al. Head-to-head comparison of in-house produced CD19 CAR-T cell in ALL and NHL patients. J Immunother Cancer. (2020) 8:e000148. doi: 10.1136/jitc-2019-000148

18. Jacoby E, Bielorai B, Avigdor A, Itzhaki O, Hutt D, Nussboim V, et al. Locally produced CD19 CAR T cells leading to clinical remissions in medullary and extramedullary relapsed acute lymphoblastic leukemia. Am J Hematol. (2018) 93:1485-92. doi: 10.1002/ajh.25274

19. Walker AJ, Majzner RG, Zhang L, Wanhainen KM, Long AH, Nguyen SM, et al. Tumor antigen and receptor densities regulate efficacy of a chimeric antigen receptor targeting anaplastic lymphoma kinase. Mol Ther. (2017) 25:2189-201. doi: 10.1016/j.ymthe.2017.06.008

20. Zhao D, Wu J, Zhao Y, Shao W, Cheng Q, Shao X, et al. Zoledronic acid inhibits TSC2-null cell tumor growth via RhoA/YAP signaling pathway in mouse models of lymphangioleiomyomatosis. Cancer Cell Int. (2020) 20:1-11. doi: 10.1186/s12935-020-1131-4

21. Santolaria T, Robard M, Leger A, Catros V, Bonneville M, Scotet E. Repeated systemic administrations of both aminobisphosphonates and human $\mathrm{V} 9 \mathrm{~V}$ $2 \mathrm{~T}$ cells efficiently control tumor development in vivo. J Immunol. (2013) 191:1993-2000. doi: 10.4049/jimmunol.1300255
22. Deniger DC, Switzer K, Mi T, Maiti S, Hurton L, Singh H, et al. Bispecific T-cells expressing polyclonal repertoire of endogenous $\gamma \delta$ T-cell receptors and introduced CD19-specific chimeric antigen receptor. Mol Ther. (2013) 21:638-47. doi: 10.1038/mt.2012.267

23. Themeli M, Kloss CC, Ciriello G, Fedorov VD, Perna F, Gonen M, et al. Generation of tumor-targeted human T lymphocytes from induced pluripotent stem cells for cancer therapy. Nat Biotechnol. (2013) 31:928-33. doi: $10.1038 /$ nbt. 2678

24. Brentjens RJ, Santos E, Nikhamin Y, Yeh R, Matsushita M, La Perle $\mathrm{K}$, et al. Genetically targeted $\mathrm{T}$ cells eradicate systemic acute lymphoblastic leukemia xenografts. Clin Cancer Res. (2007) 13:5426-35. doi: 10.1158/1078-0432.CCR-07-0674

25. Casetti R, Perretta G, Taglioni A, Mattei M, Colizzi V, Dieli F, et al. Drug-induced expansion and differentiation of $\mathrm{Vd} 9 \mathrm{Vg} 2 \mathrm{~T}$ cells in vivo: the role of exogenous IL-2. J Immunol. (2005) 175:1593-8. doi: 10.4049/jimmunol.175.3.1593

26. Pillai V, Muralidharan K, Meng W, Bagashev A, Oldridge DA, Rosenthal J, et al. CAR T-cell therapy is effective for CD19-dim B-lymphoblastic leukemia but is impacted by prior blinatumomab therapy. Blood Adv. (2019) 3:3539-49. doi: 10.1182/bloodadvances.20190 00692

27. Hartmann J, Schüßler-Lenz M, Bondanza A, Buchholz CJ. Clinical development of CAR T cells-challenges and opportunities in translating innovative treatment concepts. EMBO Mol Med. (2017) 9:e201607485. doi: $10.15252 / \mathrm{emmm} .201607485$

Conflict of Interest: The authors declare that the research was conducted in the absence of any commercial or financial relationships that could be construed as a potential conflict of interest.

Copyright (c) 2020 Rozenbaum, Meir, Aharony, Itzhaki, Schachter, Bank, Jacoby and Besser. This is an open-access article distributed under the terms of the Creative Commons Attribution License (CC BY). The use, distribution or reproduction in other forums is permitted, provided the original author(s) and the copyright owner(s) are credited and that the original publication in this journal is cited, in accordance with accepted academic practice. No use, distribution or reproduction is permitted which does not comply with these terms. 